\title{
The Design and Realization of Low Frequency Signal Receiver with High Sensitivity
}

\author{
Zhigang $\mathrm{Li}^{\mathrm{a}}$, Yuchuan Zhao ${ }^{\mathrm{b}}$ \\ College of Information and Communication Engineering of Harbin Engineering University, Harbin \\ 150001, China \\ alizhigang@hrbeu.edu.cn, ${ }^{\mathrm{b}}$ zhaoyuchuan@hrbeu.edu.cn
}

Keywords: weak signal; receiver; filter; AGC.

\begin{abstract}
This paper introduces a kind of receiver that can receive signal which weak to UV level. First, the common mode rejection for the input differential signal is necessary, and a low noise amplifier followed by. Then we should let the signal go through a Programmable filter whose center frequency, Q are all selected via pin-strapped inputs. In order to get the appropriate amplitude of the signal, an automatic gain control amplifier is needed. Last, before the signal sampled by the A/D converters, an active filter and an operational amplifier is followed by the AGC amplifier. Through the actual test, the low frequency signal receiver with high sensitivity introduced in this paper has the characteristics of high sensitivity and stability, so it can be applied in some engineering.
\end{abstract}

\section{Introduction}

Nowadays, most signals received by the antenna and sensor devices are very weak, so the extraction of weak signal from the noise outside seems more important. With the rapid development of the integrated chip, the application of some high performance chips makes the design of circuit more convenient. In this paper, a high precision instrumentation amplifier INA129 is used as the pre amplifier with low noise. And the signal will go through MAX267BCWG, the Pin Programmable Universal and Band pass filter, letting the received signal amplitude amplification to the specified range. The application of low power variable gain amplifier AD8338 makes the root mean square value of the output-signal fixed.

The Principle and Design Hardware. The system diagram is shown in Figure 1 and the digital program signal from FPGA.

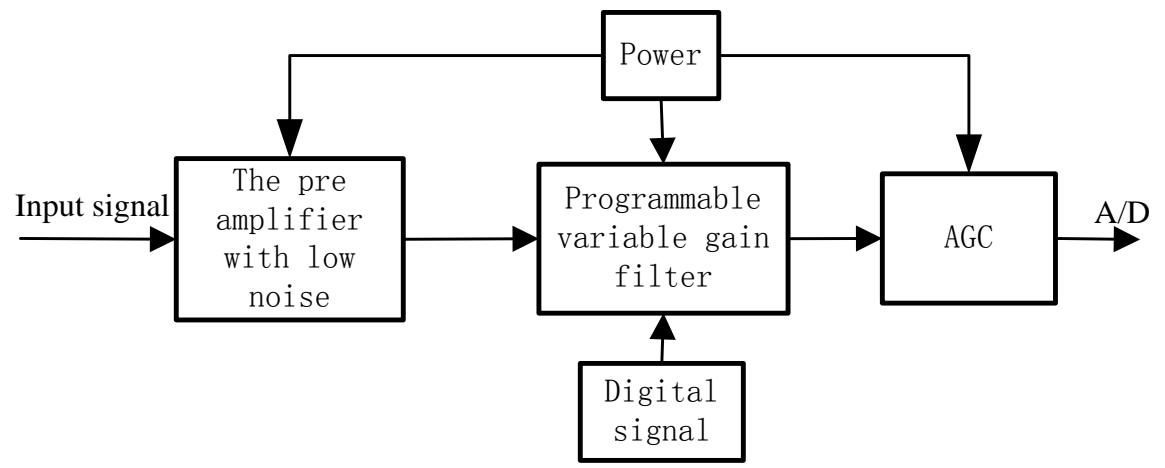

Fig.1 receiver system block diagram

The Low Noise Preamplifier. The chip INA129 is low power, general purpose instrumentation amplifiers offering excellent accuracy. A single external resistor can set any gain from 1 to 10,000 . INA129 is laser trimmed for very low offset voltage, temperature drift and high common-mode rejection. It can work normally with power supplies as low as $\pm 2.25 \mathrm{~V}$, and the quiescent current is only $700 \mu \mathrm{A}$, so it is ideal for battery operated systems. In addition, with the protection of internal input protection circuit, it can withstand up to $\pm 40 \mathrm{~V}$ and not be damaged.

In practical application, we should fully suppress the power line interference and the common-mode interference existed at the receiving terminal. The easiest way is letting the signal go 
through a Common mode inductor and a high pass filter. The high pass filter uses a passive RC network to realize, the cut-off frequency

. Because of the received signal is extremely weak, so the pre amplifier with low noise INA 129 should power alone. In order to minimize the noise impact on the post stage circuit, the gain should not be too large. The specific circuit is shown in figure 2:

The gain of INA129 $G=1+\frac{50 K \Omega}{R_{G}}$.

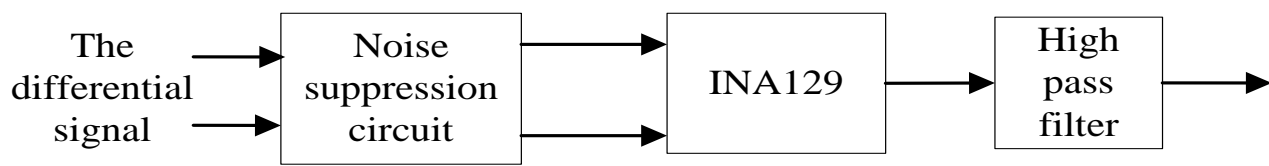

Fig.2 Schematic diagram of low noise preamplifier

Programmable Filter. MAX267BCWG is a programmable precision band pass filter which has 32 level mode for the center frequency change and 128 kinds of $\mathrm{Q}$ value choice. Each MAX267BCWG contains two independent second-order filters which can be directly applied in series. The gain of each order filter is only related Q value. In addition, each chip has an uncommitted op-amp. We can change the ratio of input resistor and feedback resistor to control the gain. So the control of the gain is very convenient. In this design, we cascade two second-order filters, the signal goes through the uncommitted amplifier first and then a four order filter followed by. A total of three pieces chips are used to form a twelve-order filter and the gain of each chip is 10 times. The specific circuit is shown in figure 3. As can be seen from the chart, some of the pins which control the $Q$ value and the center frequency connect to power supply directly to force its voltage fixed. Other control pins and the clock signal is controlled by FPGA. In order to avoid the interference between digital signal and analog signal, an optical coupler will be used to isolation control. At the same time, we also need to make the isolation of analog ground and digital ground to suppress the interference from each other.

The gain of each piece MAX267BCWG: $A=\frac{R_{1}}{R_{2}} Q^{2}$

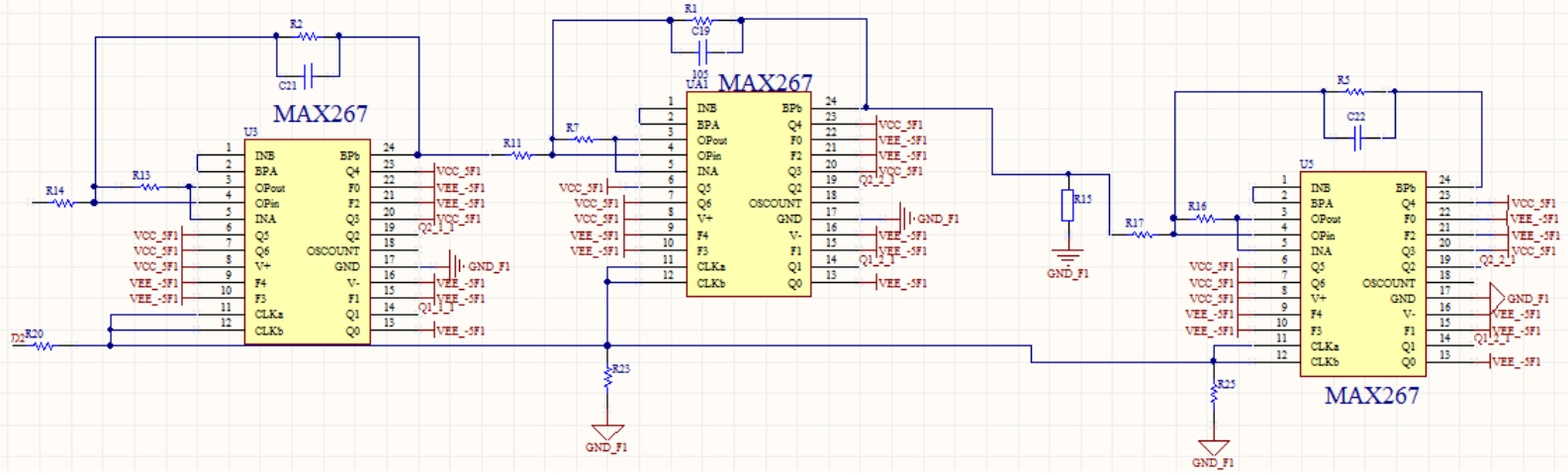

Fig.3 the band pass filter circuit based on MAX267BCWG

Automatic Gain Control Circuit. Processing by the low noise preamplifier and the twelve-order filter, the signal will be sent to the AGC circuit. Here, a Low Power, $18 \mathrm{MHz}$ Variable Gain Amplifier AD8338 which will be used. While the inputs are differential, the device can also be driven with a single-ended source if required. An additional circuit can be used to implement the offset correction and automatic gain control function. The AD8338 includes an internal offset correction circuit and connecting a capacitor between Pin OFSN and Pin VREF enables the offset correction circuit. We can change the voltage on the VAGC pin to keep the root-mean-squire value (rms) constant. When the voltage of VAGC is set to $1.5 \mathrm{~V}$, the target output voltage is minimal. The specific calculation formulas are as follows:

$$
\begin{array}{ll}
V_{\text {ORИS }}=1.7 \times V A G C-2.264 & V A G C \geq 1.5 V \\
V_{\text {ORИS }}=-1.7 \times V A G C+2.864 & V A G C \leq 1.5 V
\end{array}
$$


The specific circuit is shown in Figure 4.

From the graph we can see that the device is driven with a single-ended source. The other differential input pin connects a resistor and a capacitor to the ground. This design uses INPR and INMR as the input pins and the gain range controlled by the two resistors front of each input pin. If the two resistors are valued to 0 , the gain adjustment range will be $0 \mathrm{~dB}$ to $80 \mathrm{~dB}$. The RMS value of output can be adjusted by changing the ratio of R3 and R6. In this design, two maximum reference voltages are provided, the $1.5 \mathrm{~V}$ fixed output of pin 16 and the $5 \mathrm{~V}$ from the power VCC_5A1. The out0put of AD8338 has a DC component of $1.5 \mathrm{~V}$, so a high pass filter followed is necessary. Through the actual measurement, a constant output can be obtained when the input voltage is at the range of $3 \mathrm{mV}$ to the power supply voltage.

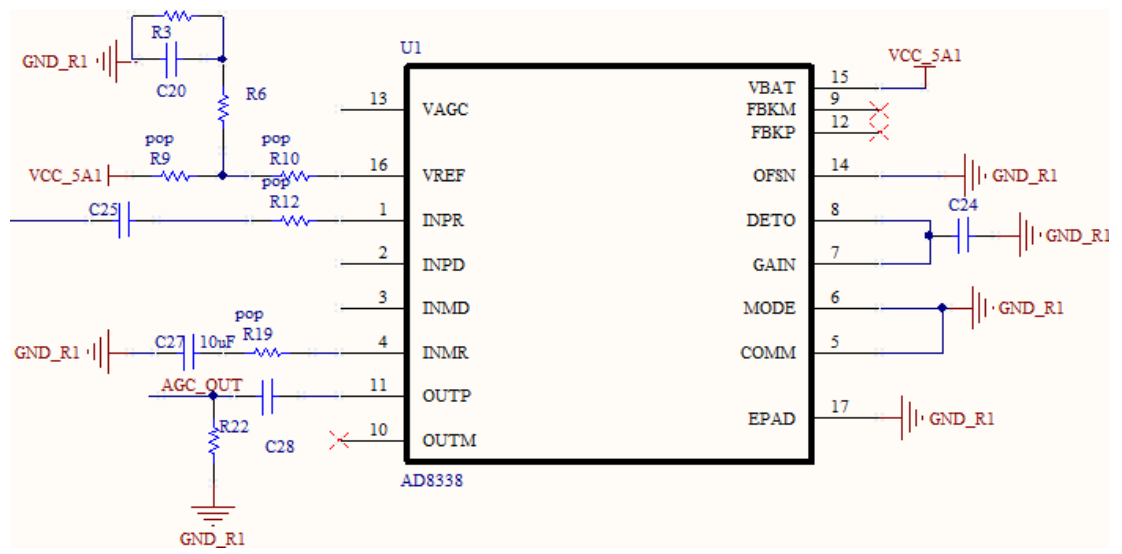

Fig.4 automatic gain control circuit

The Circuit before A/D. As shown in Figure 5, in order to make the voltage of output signal suitable for sampling and processing by A/D, an operational amplifier LT1028 is followed and amplifies the signal to the target range. The gain of $\mathrm{EF1} 28$ is . The second order active high pass filter composed of opa211 is mainly used to suppress the high frequency noise out of band. At the end of the system, there is a voltage following circuit. The circuit mainly plays a role of buffering and reduces the effect of the circuit before $\mathrm{A} / \mathrm{D}$ on the sampling precision. Making the sampling value is as close as possible to the real value.

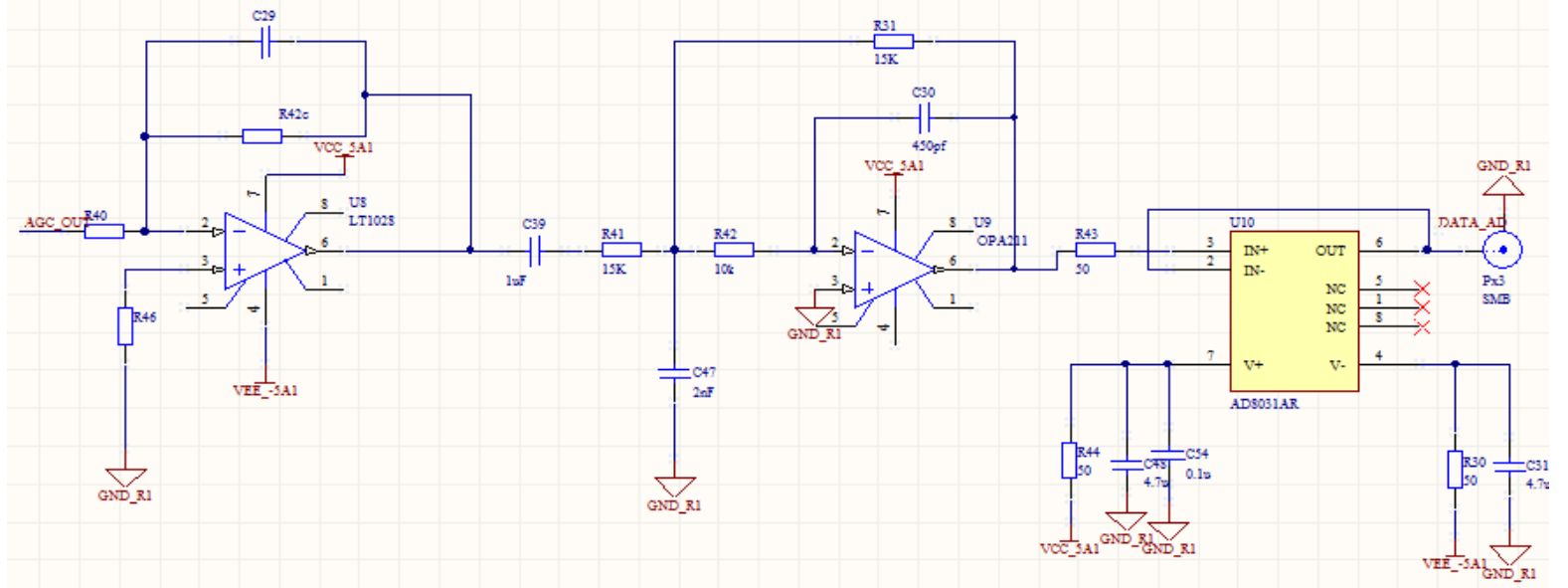

Fig. 5 the circuit before A/D

System Test. The hardware of receiver is shown in figure 6 . The waveform of the MAX267BCWG's output and the final output were observed at differential input signal of $10 \mathrm{uV}$. The results are shown in Figure 7 and Figure 8. As can be seen from the graphs, the waveforms of each output are clear and stable. We made a study on the output signals' spectrum of the MAX267BCWG and the AGC. There are some components of high frequency noise existed in the signal. After the treatment of the second order band pass filter, the effect of final output is obviously improved. 


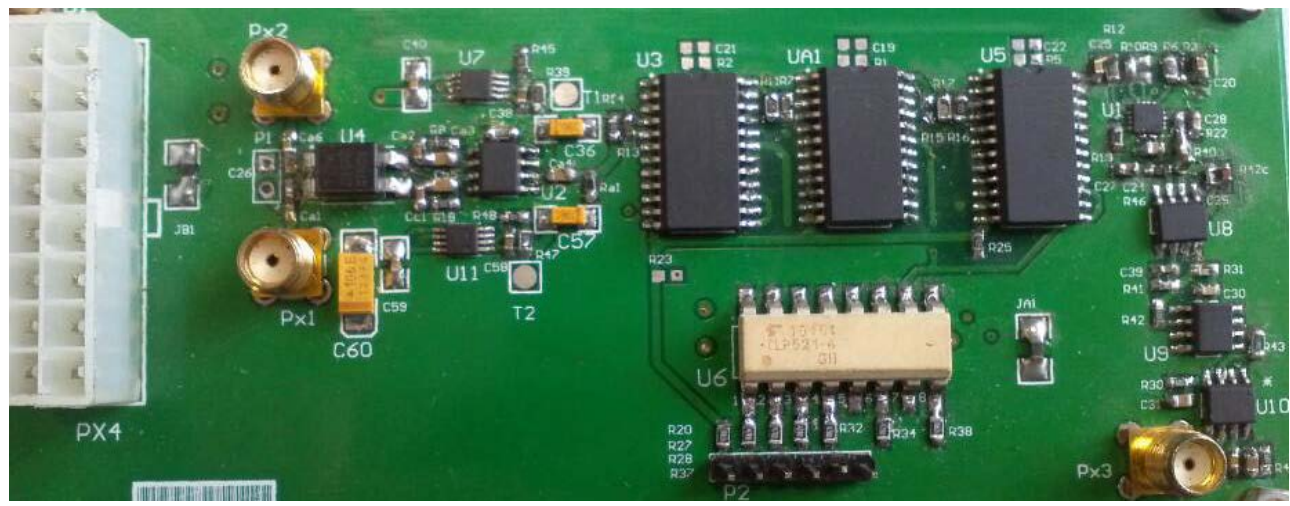

Fig.6 The hardware of receiver

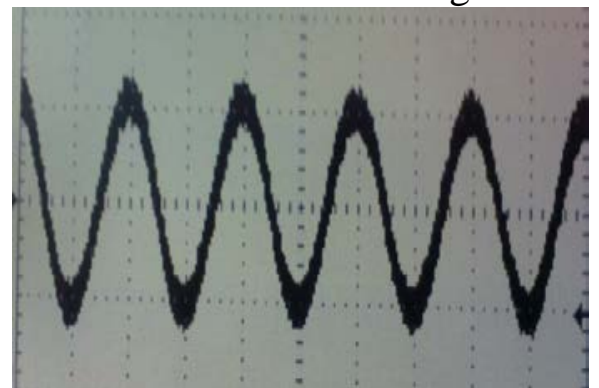

Fig.7 The waveform of MAX267BCWG

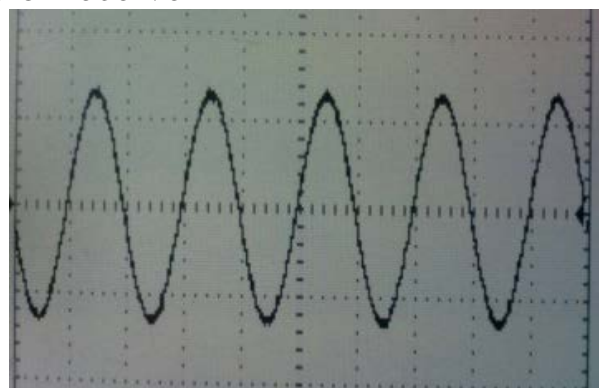

Fig.8 The waveform of the final output

\section{Summary}

This paper introduces a design scheme of low frequency signal receiver with high sensitivity. We verified the stability and feasibility of the proposed scheme though the actually test. The receiver has a wide dynamic range of the received signal's amplitude and the sensitivity of it is very high. So it may be suitable in some sensors or wireless communication systems and has some practical application value.

\section{Acknowledgments}

This paper is funded by the International Exchange Program of Harbin Engineering University for Innovation-oriented Talents Cultivation, International Science \& Technology Cooperation Program of China (2014 DFR10240), National Natural Science Foundation of China (612111070), China Postdoctoral Science Foundation (2013T60346), Harbin science and technology research projects (2013AE1BE003), Heilongjiang province natural science foundation projects (F201344).

\section{References}

[1] Hong Xie, Basis of Analogue Electronic Technique [M]. Harbin Engineering University press, 2008,228-230P

[2] High performance microcontroller and analog devices of TI applicate in the college and Selection Guide [M].TI (Shanghai) University Planning Department, 2012.6

[3] Yindong Xiao, Hui Zhao, Houjun Wang. Design of weak signal detection circuit of pre filter based on phase-locked amplification. Measurement and control technology 6 (2007)

[4] Jeffrey R.Riskin.IC The instrumentation amplifier User Guide.AN-244 Application notes.

[5] Henry J.Zhang. The basic concept of linear regulator and switching power supply. Application Note 140. (2013) 